\title{
Outcomes of endoscopic sinus surgery for sinusitis-induced intracranial abscess in patients undergoing neurosurgery
}

\author{
Megumi Koizumi, MD, ${ }^{1,2}$ Miho Ishimaru, MPH, ${ }^{1}$ Hiroki Matsui, MPH, ${ }^{1}$ Kiyohide Fushimi, MD, PhD, ${ }^{3}$ \\ Tatsuya Yamasoba, MD, PhD, ${ }^{2}$ and Hideo Yasunaga, MD, PhD'
}

1Department of Clinical Epidemiology and Health Economics, School of Public Health; ${ }^{2}$ Department of Otolaryngology and Head and Neck Surgery, Faculty of Medicine, The University of Tokyo; and ${ }^{3}$ Department of Health Policy and Informatics, Tokyo Medical and Dental University Graduate School of Medicine, Tokyo, Japan

\begin{abstract}
OBJECTIVE Although sinusitis-induced intracranial complications rarely occur in the current era of antibiotics, they can induce neurological symptoms or death. The authors of this study investigated the association between endoscopic sinus surgery (ESS) and outcomes in patients who had undergone neurosurgical procedures for sinusitis-induced intracranial abscess.

METHODS The authors obtained data on patients with sinusitis-induced intracranial abscess from the Japanese Diagnosis Procedure Combination inpatient data for the period from 2010 to 2017 . They excluded patients with fungal sinusitis, orbital complications, immunodeficiency, and malignant disease. They also excluded patients who had received antifungal agents, chemotherapy, immunosuppressants, and antidiabetic drugs. Eligible patients were divided into those with and those without neurosurgical procedures. Propensity score-adjusted regression analyses were performed to examine the association between ESS within the same hospitalization and outcomes (mortality, blood transfusion, readmission, revision neurosurgery, and length of stay).
\end{abstract}

RESULTS Of the 552 potentially eligible patients, 255 were treated with neurosurgical procedures, including 104 who underwent ESS within the same hospitalization and 151 who did not. ESS was not significantly associated with mortality (OR 0.54, 95\% Cl 0.05-5.81, $p=0.61$ ), blood transfusion (OR 1.95, 95\% Cl 0.84-4.51, p = 0.12), readmission (OR 0.86, $95 \% \mathrm{Cl} 0.34-2.16, \mathrm{p}=0.75$ ), revision neurosurgery (OR 0.65, 95\% $\mathrm{Cl} 0.24-1.74, \mathrm{p}=0.39$ ), or length of stay (percent difference $-10.8 \%, 95 \% \mathrm{Cl}-24.4 \%$ to $5.1 \%, p=0.18$ ).

CONCLUSIONS The present study suggests that ESS may not have significant benefits with respect to reducing mortality, blood transfusion, readmission, revision neurosurgery, or length of stay.

https://thejns.org/doi/abs/10.3171/2019.5.FOCUS1947

KEYWORDS intracranial complication; endoscopic sinus surgery; mortality; neurosurgical reoperation; retrospective database study; propensity score

$\mathrm{A}$ CUTE and chronic bacterial sinusitis are common in both adult and pediatric patients. However, serious intracranial complications of sinusitis rarely occur. Previous studies have shown that the incidence of intracranial complications ranges from $3.7 \%$ to $14.6 \% \%^{1,4,13}$ and that the mortality rate ranges from $0 \%$ to $20 \% .2,5,6,7,12,17$ Potential intracranial complications include brain abscess, epidural abscess, subdural abscess, meningitis, cerebral venous sinus thrombosis, and cerebritis..$^{10,18}$ Long-term neurological deficits, such as hemiparesis, aphasia, epilepsy, hydrocephalus, and visual deficits, occur in 13\%-35\% of patients with bacterial sinusitis. ${ }^{6,79}$ Treatments for these life-threatening conditions include intensive intravenous antibiotic therapy, neurosurgical procedures to remove central nervous system abscesses, and sinus surgery to remove paranasal sinus abscesses. ${ }^{10}$

Most patients with sinusitis-induced intracranial complications reportedly undergo sinus surgery. A previous study showed that endoscopic sinus surgery (ESS) has a key role in controlling infection, ${ }^{8}$ while another study indicated that the role of ESS in intracranial complications of sinusitis is not clear. ${ }^{2}$ ESS for acute sinusitis can be chal-

ABBREVIATIONS BMI = body mass index; $\mathrm{CCI}=$ Charlson Comorbidity Index; ESS = endoscopic sinus surgery; JCS = Japan Coma Scale ACCOMPANYING EDITORIAL DOI: 10.3171/2019.5.FOCUS19374.

SUBMITTED January 22, 2019. ACCEPTED May 10, 2019.

INCLUDE WHEN CITING DOI: 10.3171/2019.5.FOCUS1947. 


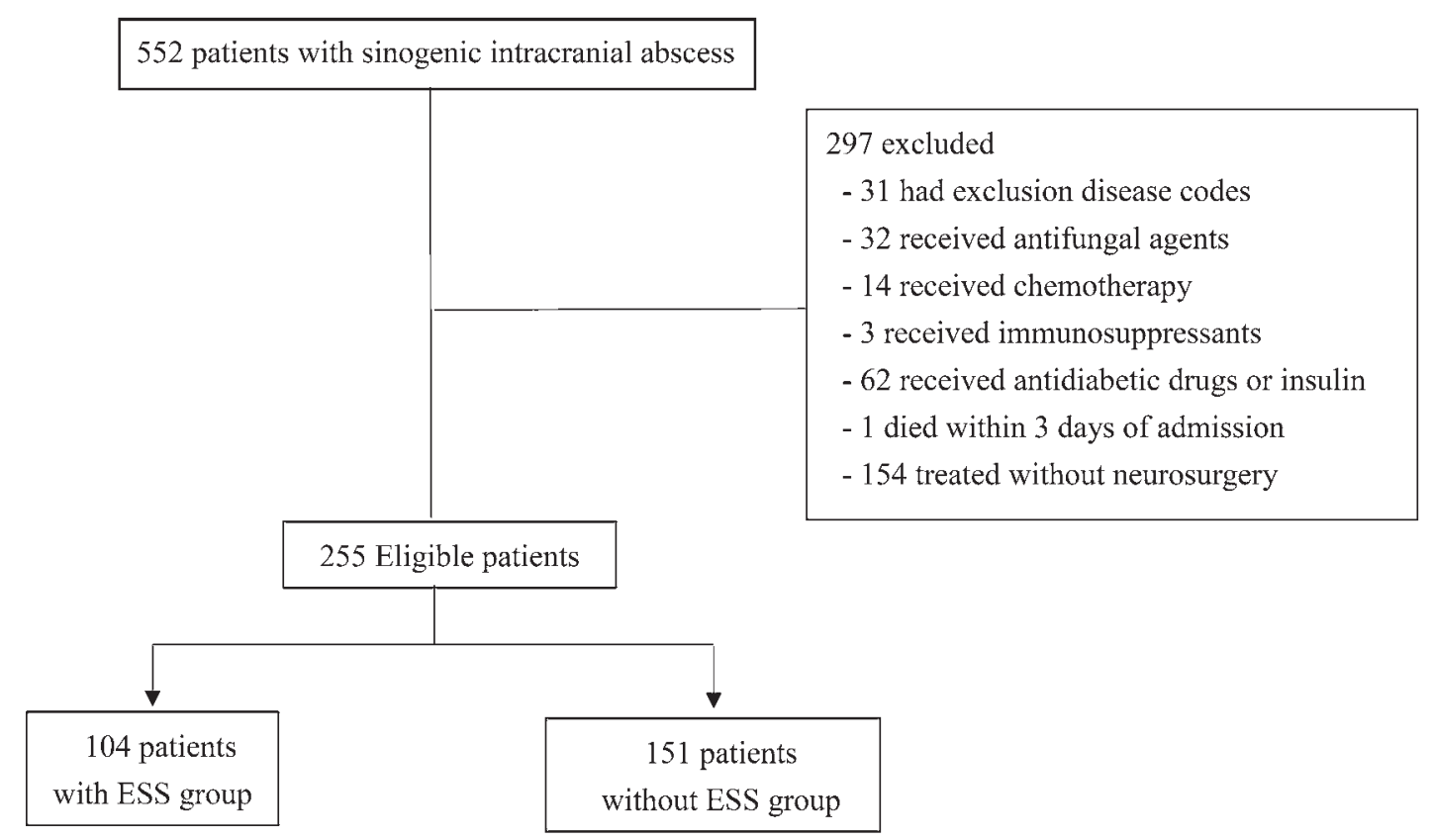

FIG. 1. Flowchart of the patient selection process.

lenging because of bleeding caused by mucosal inflammation. ${ }^{2}$ With respect to neurosurgical procedures, previous studies have shown that patients with meningitis and a small intracranial abscess $(<1 \mathrm{~cm})$ without significant mass effect can be managed by antibiotics without neurosurgical procedures. ${ }^{2,18}$

A systematic review involving 16 studies and 180 pediatric patients (age $<18$ years) did not reveal the outcomes of ESS. ${ }^{10}$ Large studies are necessary to evaluate the role of ESS in the intracranial complications of sinusitis. A practical guideline for the management of acute rhinosinusitis in Japan ${ }^{16}$ states that treatments for rhinosinusitisinduced orbital or intracranial complications are slightly different among hospitals and countries. In this Japanese guideline, the level of evidence of treatment for orbital and intracranial complications is III (at least one well-designed nonexperimental descriptive study).

In the present retrospective observational study, we investigated the outcomes of ESS in terms of mortality, blood transfusion, readmission, revision neurosurgery, and length of stay and examined the association between ESS within the same hospitalization and these outcomes using a national inpatient database in Japan.

\section{Methods \\ Data Source}

We used the Diagnosis Procedure Combination database, which is a national inpatient database in Japan. The database includes administrative claims data and discharge abstract data. The database collects information from more than 1000 participating acute-care hospitals across Japan and includes approximately 7 million patients per year. The database contains information regarding patient age, sex, height, weight, smoking status, state of consciousness at admission, length of stay, discharge status, main diagnosis at admission, comorbidities at admission, complications after admission, dates of admission and operation, surgical procedures, drugs and devices used, and hospital codes.

Because of the anonymous nature of the data, the requirement for informed consent was waived. Study approval was obtained from the institutional review board of The University of Tokyo.

\section{Patient Sample}

We included patients who had had acute or chronic sinusitis and a brain, epidural, or subdural abscess in the period from July 2010 to March 2017. We obtained data on patients using the International Statistical Classification of Diseases and Related Health Problems, 10th Revision (ICD-10) codes of brain abscess (G060), epidural or subdural abscess (G062), acute sinusitis (J01), and chronic sinusitis (J32). We excluded patients with codes for an orbital abscess (H050), fungal sinusitis (B49), immunodeficiency (D70, D71, D76, D80-D84), acquired immune deficiency syndrome (B20-B24), and malignant lymphoma or leukemia (C81-C96), as well as patients who had received antifungal agents, antineoplastic agents and immunosuppressants, and antidiabetic drugs or insulin because these patients were likely to undergo ESS. We also excluded patients who had died within 3 days of admission to account for immortal time bias. We selected patients who had undergone neurosurgery and divided them into those who did and those who did not undergo ESS within the same hospitalization.

\section{Variables}

We obtained the following patient data from the data- 
TABLE 1. Summary of patient characteristics

\begin{tabular}{|c|c|c|c|}
\hline Variable & ESS Group & Control Group & $p$ Value \\
\hline No. of patients & 104 & 151 & \\
\hline Sex & & & 0.62 \\
\hline Male & $76(73.1)$ & $106(70.2)$ & \\
\hline Female & $28(26.9)$ & $45(29.8)$ & \\
\hline Age in yrs & & & 0.07 \\
\hline $0-19$ & $28(26.9)$ & $24(15.9)$ & \\
\hline $20-39$ & $20(19.2)$ & $20(13.2)$ & \\
\hline $40-59$ & $24(23.1)$ & $39(25.8)$ & \\
\hline $60-79$ & $28(26.9)$ & $57(37.7)$ & \\
\hline$\geq 80$ & $4(3.8)$ & $11(7.3)$ & \\
\hline $\mathrm{BMI}$ in $\mathrm{kg} / \mathrm{m}^{2}$ & & & 0.20 \\
\hline$<18.5$ & $29(27.9)$ & 27 (17.9) & \\
\hline $18.5-24.9$ & $50(48.1)$ & $83(55.0)$ & \\
\hline $25.0-29.9$ & $15(14.4)$ & $24(15.9)$ & \\
\hline$\geq 30.0$ & $6(5.8)$ & $5(3.3)$ & \\
\hline Missing data & $4(3.8)$ & $12(7.9)$ & \\
\hline Smoking & & & 0.33 \\
\hline Nonsmoker & $58(55.8)$ & $84(55.6)$ & \\
\hline Current/past smoker & $26(25.0)$ & 47 (31.1) & \\
\hline Unspecified & $20(19.2)$ & $20(13.2)$ & \\
\hline $\mathrm{CCl}$ & & & 0.35 \\
\hline 0 & $85(81.7)$ & $130(86.1)$ & \\
\hline$\geq 1$ & $19(18.3)$ & $21(13.9)$ & \\
\hline \multicolumn{4}{|l|}{ JCS score } \\
\hline 0 & $52(50.0)$ & $83(55.0)$ & 0.63 \\
\hline $1-3$ & $34(32.7)$ & $50(33.1)$ & \\
\hline $10-30$ & $13(12.5)$ & $14(9.3)$ & \\
\hline $100-300$ & $5(4.8)$ & $4(2.6)$ & \\
\hline Type of hospital & & & 0.05 \\
\hline Academic & $30(28.8)$ & $28(18.5)$ & \\
\hline Nonacademic & 74 (71.2) & $123(81.5)$ & \\
\hline
\end{tabular}

Data are presented as number (\%).

base: sex, age ( $\leq 19,20-39,40-59,60-79$, or $\geq 80$ years), body mass index (BMI; < 18.5, 18.5-22.9, 23.0-24.9, $25.0-29.9$, or $\geq 30.0 \mathrm{~kg} / \mathrm{m}^{2}$ ), smoking status (nonsmoker or current/past smoker), Charlson Comorbidity Index (CCI; 0 or $\geq 1$ ), fiscal year of admission (2010, 2011, 2012, 2013, 2014, 2015, or 2016), Japan Coma Scale (JCS) score on admission $(0,1-3,10-30$, or 100-300), and type of hospital (academic or nonacademic). The JCS is a one-axis coma scale. ${ }^{11}$ JCS scores of $0,1-3,10-30$, and 100-300 represent alert consciousness, drowsiness, somnolence, and coma, respectively. ${ }^{14}$ We categorized BMI according to the definition established by the World Health Organization. ${ }^{15}$

The outcomes included in-hospital mortality, blood transfusion, readmission, reoperation of neurosurgical procedures, and length of stay.

\section{Statistical Method}

We compared the proportions of in-hospital deaths, re-
TABLE 2. Factors in crude comparison

\begin{tabular}{lccc}
\hline \multicolumn{1}{c}{ Factor } & ESS Group & Control Group & p Value \\
\hline Mortality & $1(1.0)$ & $3(2.0)$ & 0.92 \\
\hline Blood transfusion & $16(15.4)$ & $12(7.9)$ & 0.10 \\
\hline Readmission & $8(7.7)$ & $18(11.9)$ & 0.38 \\
\hline Revision neurosurgery & $9(8.7)$ & $12(7.9)$ & 1.01 \\
\hline Length of stay & $45.1 \pm 28.3$ & $49.9 \pm 34.3$ & 0.44 \\
\hline
\end{tabular}

Data are presented as number (\%) or mean \pm standard deviation.

admissions, blood transfusions, or revision neurosurgery between the two groups using the chi-square test. We also compared the length of stay between the two groups using the Mann-Whitney U-test. We calculated propensity scores using a logistic regression analysis to adjust for the characteristics of patients. We estimated propensity scores using the following eight variables: sex, age, BMI, smoking status, CCI, fiscal year, JCS score, and type of hospital. We calculated the C-statistics and then performed logistic regression analyses to evaluate the association of ESS with in-hospital mortality, blood transfusion, readmission, or revision neurosurgery with adjustment for propensity scores. We also performed a multiple linear regression analysis with propensity score adjustment to assess the association between ESS and length of stay. Length of stay was log transformed. For a sensitivity analysis, we investigated the association between the outcomes and ESS performed within 3 days of admission using the same analyses described above. We performed subgroup analyses by stratifying patients into a brain abscess group and subdural/epidural abscess group. These analyses were performed using the same methods as described above. In these subgroup analyses, we excluded patients who had both diagnoses. The threshold for significance was $p<0.05$. We used Stata version 15.0 (StataCorp LLC) for all analyses.

\section{Results}

We identified 552 patients with a main diagnosis of sinusitis-induced brain abscess (G060) or epidural/subdural abscess (G062) in the period from July 1, 2010, to March 31,2017 . Figure 1 is a flowchart of the patient selection process. Of the 255 eligible patients, 104 underwent ESS and 151 did not. Of the 255 patients, 247 received intravenous antibiotics, 3 took antibiotic tablets, and 5 received no antibiotics. The median interval from admission to starting antibiotics was 1 day (IQR 1-2 days). Neurosurgical procedures included drainage alone (119 patients), craniotomy alone (117), and both (19). The median interval from admission to starting ESS was 3 days (IQR 2-8 days).

Table 1 shows the characteristics of the patients. Those with a sinusitis-induced intracranial abscess were more frequently male and elderly. There was no significant difference in the characteristics of patients between the ESS and control groups. There was a nonsignificant difference in in-hospital mortality, blood transfusion, readmission, revision neurosurgery, and length of stay between the two groups in the crude data comparisons (Table 2).

In the propensity score-adjusted analyses, the C-sta- 
TABLE 3. Multivariable logistic regression and multivariable linear regression for propensity score-adjusted analysis

\begin{tabular}{ccc}
\hline Variable & OR $(95 \% \mathrm{Cl})$ & $\mathrm{p} \mathrm{Value}$ \\
\hline Mortality & & \\
\hline ESS group & $0.54(0.05-5.80)$ & 0.61 \\
\hline Control group & Reference & \\
\hline Blood transfusion & & 0.12 \\
\hline ESS group & $1.95(0.84-4.51)$ & \\
\hline Control group & Reference & \\
\hline Readmission & & \\
\hline ESS group & $0.86(0.34-2.16)$ & 0.39 \\
\hline Control group & Reference \\
\hline Revision neurosurgery & & \\
\hline ESS group & $0.65(0.24-1.74)$ & \\
\hline Control group & Reference & \\
\hline Length of stay & & \\
\hline ESS group & $-10.8 \%{ }^{*}(-24.4 \%$ to $5.1 \%)$ & \\
\hline Control group & Reference \\
\hline
\end{tabular}

Propensity scores were calculated with consideration of sex, age, BMI, smoking status, comorbidity at admission, fiscal year of admission, JCS score, and type of hospital.

${ }^{*}$ Percent difference.

tistic was 0.7. Logistic regression analyses with propensity score adjustment showed no significant association of ESS with mortality (OR 0.54,95\% CI 0.05-5.80, $\mathrm{p}=0.61$ ), blood transfusion (OR 1.95, 95\% CI 0.84-4.51, $\mathrm{p}=0.12$ ), readmission (OR $0.86,95 \%$ CI $0.34-2.16, \mathrm{p}=0.75)$, or re- vision neurosurgical procedures (OR $0.65,95 \%$ CI 0.24 $1.74, p=0.39$ ). Multiple linear regression after propensity score adjustment showed that ESS was not significantly associated with length of stay (percent difference $-10.8 \%$, $95 \%$ CI $-24.4 \%$ to $5.1 \%, \mathrm{p}=0.18$; Table 3 ).

The sensitivity analyses showed trends similar to those for the main analyses. The subgroup analyses also showed trends similar to those for the main analyses (Table 4).

\section{Discussion}

There was a nonsignificant association between ESS for the treatment of sinusitis-induced intracranial abscess and outcomes in patients who underwent neurosurgery. This may be explained by how inflammation extends to the central nervous system. Infection of a sinus spreads mostly by progression of septic thrombi through the valveless diploic veins of the skull base that penetrate the dura mater. Direct extension via osteomyelitis of the sinus walls or bony defects (congenital or posttraumatic) is uncommon.,18 Thus, the effect of drainage from the sinus may be limited. The present study showed no significant benefit of ESS in terms of reducing in-hospital mortality, blood transfusion, readmission, revision neurosurgery, or length of stay. This finding will help physicians make treatment decisions for patients with a sinusitis-induced intracranial abscess.

A previous study showed that early ESS tended to reduce revision neurosurgery. ${ }^{9}$ In the present study, approximately $80 \%$ of ESS procedures were performed within 8 days of admission. We, therefore, assessed ESS within 3 days of admission as a sensitivity analysis. The results were similar to those in the main analyses. These data indicate the robustness of our results.

We found that male and elderly patients accounted for

TABLE 4. Multivariable logistic regression and multivariable linear regression for subgroup analysis

\begin{tabular}{|c|c|c|c|c|}
\hline \multirow[b]{2}{*}{ Variable } & \multicolumn{2}{|c|}{ Brain Abscess Group } & \multicolumn{2}{|c|}{ Subdural/Epidural Abscess Group } \\
\hline & OR (95\% Cl) & $p$ Value & OR (95\% Cl) & $\mathrm{p}$ Value \\
\hline \multicolumn{5}{|l|}{ Mortality* } \\
\hline ESS group & - & & $1.59(0.07-35.2)$ & 0.77 \\
\hline Control group & - & & Reference & \\
\hline \multicolumn{5}{|l|}{ Blood transfusion } \\
\hline ESS group & $2.50(0.68-9.22)$ & 0.17 & $1.71(0.51-5.72)$ & 0.39 \\
\hline Control group & Reference & & Reference & \\
\hline \multicolumn{5}{|l|}{ Readmission } \\
\hline ESS group & $1.45(0.37-5.70)$ & 0.60 & $0.71(0.19-2.74)$ & 0.62 \\
\hline Control group & Reference & & Reference & \\
\hline \multicolumn{5}{|c|}{ Revision neurosurgery } \\
\hline ESS group & $0.83(0.22-3.19)$ & 0.79 & $0.50(0.10-2.52)$ & 0.40 \\
\hline Control group & Reference & & Reference & \\
\hline \multicolumn{5}{|l|}{ Length of stay } \\
\hline ESS group & $-12.5 \% \dagger(-29.7 \%$ to $8.9 \%)$ & 0.23 & $-5.6 \% \dagger(-28.1 \%$ to $23.6 \%)$ & 0.67 \\
\hline Control group & Reference & & Reference & \\
\hline
\end{tabular}

Propensity scores were calculated with consideration of sex, age, BMI, smoking status, comorbidity at admission, fiscal year of admission, JCS score, and type of hospital.

${ }^{*}$ Mortality was $0 \%$ in the ESS group within the brain access group.

$\dagger$ Percent difference. 
the highest proportion of patients in this study. Male patients have been dominant in many previous studies, and our results are consistent with those findings. Several studies have shown that sinusitis-induced intracranial abscesses occur more commonly among adolescents and young adults in their 20s and 30s., $32,17,18$ Our results are inconsistent with those of previous studies; we found that abscesses occur at any age, although the reason remains unclear.

The main strength of this study is the large number of patients; previous studies have evaluated fewer than 100 patients. Another strength is our inclusion of patients who did and who did not undergo ESS, whereas most previous studies have included only patients who underwent ESS without a control group. The present study showed similar trends in the subgroup analyses and sensitivity analyses. We consider that the robustness of our results is also a strength of our study.

\section{Study Limitations}

We acknowledge three main limitations to this study. First, we could not obtain information on the size of the intracranial abscess or severity of sinusitis. Second, we could not identify patients with direct extension of inflammation via a bony defect near the intracranial abscess because of the lack of imaging data. Third, information on the causative microorganism was lacking. These limitations have the potential to impact the treatment decisionmaking process; however, the patients' characteristics and backgrounds were not significantly different. We, therefore, consider these impacts to be small.

\section{Conclusions}

Our analysis suggests that ESS may not always be necessary for patients with a sinusitis-induced intracranial abscess requiring neurosurgery.

\section{Acknowledgments}

This work was supported by grants from the Ministry of Health, Labour and Welfare, Japan (H30-Policy-Designated-004 and H29-ICT-General-004) and the Ministry of Education, Culture, Sports, Science and Technology, Japan (17H04141).

\section{References}

1. Clayman GL, Adams GL, Paugh DR, Koopmann CF Jr: Intracranial complications of paranasal sinusitis: a combined institutional review. Laryngoscope 101:234-239, 1991

2. DelGaudio JM, Evans SH, Sobol SE, Parikh SL: Intracranial complications of sinusitis: what is the role of endoscopic sinus surgery in the acute setting. Am J Otolaryngol 31:2528,2010

3. Fountas KN, Duwayri Y, Kapsalaki E, Dimopoulos VG, Johnston KW, Peppard SB, et al: Epidural intracranial abscess as a complication of frontal sinusitis: case report and review of the literature. South Med J 97:279-283, 2004

4. Gallagher RM, Gross CW, Phillips CD: Suppurative intracranial complications of sinusitis. Laryngoscope 108:16351642,1998

5. Garin A, Thierry B, Leboulanger N, Blauwblomme T, Grevent D, Blanot S, et al: Pediatric sinogenic epidural and subdural empyema: the role of endoscopic sinus surgery. Int J Pediatr Otorhinolaryngol 79:1752-1760, 2015
6. Germiller JA, Monin DL, Sparano AM, Tom LW: Intracranial complications of sinusitis in children and adolescents and their outcomes. Arch Otolaryngol Head Neck Surg 132:969-976, 2006

7. Gitomer SA, Zhang W, Marquez L, Chandy BM: Reducing surgical revision in intracranial complications of pediatric acute sinusitis. Otolaryngol Head Neck Surg 159:359-364, 2018

8. Hibi A, Amakusa Y: Intracranial subdural abscess with polymicrobial infections due to frontal sinusitis in an adolescent: life-threatening complication of a common disease. Clin Case Rep 6:516-521, 2018

9. Jones NS, Walker JL, Bassi S, Jones T, Punt J: The intracranial complications of rhinosinusitis: can they be prevented? Laryngoscope 112:59-63, 2002

10. Patel NA, Garber D, Hu S, Kamat A: Systematic review and case report: Intracranial complications of pediatric sinusitis. Int J Pediatr Otorhinolaryngol 86:200-212, 2016

11. Shigematsu K, Nakano H, Watanabe Y: The eye response test alone is sufficient to predict stroke outcome-reintroduction of Japan Coma Scale: a cohort study. BMJ Open 3:e002736, 2013

12. Szyfter W, Bartochowska A, Borucki Ł, Maciejewski A, Kruk-Zagajewska A: Simultaneous treatment of intracranial complications of paranasal sinusitis. Eur Arch Otorhinolaryngol 275:1165-1173, 2018

13. Tandon S, Beasley N, Swift AC: Changing trends in intracranial abscesses secondary to ear and sinus disease. J Laryngol Otol 123:283-288, 2009

14. Wada T, Yasunaga H, Doi K, Matsui H, Fushimi K, Kitsuta Y, et al: Relationship between hospital volume and outcomes in patients with traumatic brain injury: A retrospective observational study using a national inpatient database in Japan. Injury 48:1423-1431, 2017

15. World Health Organization: Body mass index-BMI. World Health Organization. (http://www.euro.who.int/en/healthtopics/disease-prevention/nutrition/a-healthy-lifestyle/bodymass-index-bmi) [Accessed January 25, 2019]

16. Yamanaka N, Iino Y, Uno Y, Kudo F, Kurono Y, Suzaki H, et al: Practical guideline for management of acute rhinosinusitis in Japan. Jpn J Rhinol 49:143-104, 2010 (Japanese)

17. Yamasoba T, Kikuchi S: Intracranial complications resulting from frontal pyocele: case presentation and review of experience in Japan. Head Neck 15:450-454, 1993

18. Ziegler A, Patadia M, Stankiewicz J: Neurological complications of acute and Chronic sinusitis. Curr Neurol Neurosci Rep 18:5, 2018

\section{Disclosures}

The authors report no conflict of interest concerning the materials or methods used in this study or the findings specified in this paper.

\section{Author Contributions}

Conception and design: Koizumi. Acquisition of data: Koizumi, Ishimaru, Matsui, Fushimi, Yasunaga. Analysis and interpretation of data: Koizumi, Ishimaru, Yamasoba, Yasunaga. Drafting the article: Koizumi, Yasunaga. Critically revising the article: Yamasoba, Yasunaga. Approved the final version of the manuscript on behalf of all authors: Koizumi. Statistical analysis: Koizumi, Ishimaru, Yasunaga. Administrative/technical/material support: Ishimaru, Matsui, Fushimi, Yamasoba, Yasunaga. Study supervision: Yamasoba, Yasunaga.

\section{Correspondence}

Megumi Koizumi: School of Public Health, The University of Tokyo, Japan.imegumi-zao@umin.ac.jp. 